\title{
YAG laser iridotomy treatment for primary angle closure in east Asian eyes
}

Department of Epidemiology and International Eye Health, Institute of Ophthalmology, University College, London, UK W P Nolan P J Foster J G Devereux G J Johnson

Department of Ophthalmology, Medical University Central Hospital, Ulaanbaatar, Mongolia D Uranchimeg

G J Johnson

J Baasanhu

Correspondence to: Gordon J Johnson, International Centre for Eye Health, Institute of Ophthalmology, Bath Street, London EC1V 9EL, UK w.nolan@ucl.ac.uk

Accepted for publication 3 May 2000

Winifred P Nolan, Paul J Foster, Joe G Devereux, Davaatseren Uranchimeg, Gordon J Johnson, Jamyanjav Baasanhu

\begin{abstract}
Aim-To assess the efficacy of Nd:YAG laser iridotomy as initial treatment for primary angle closure in a community setting in rural Mongolia.

Methods-Subjects with occludable drainage angles in two glaucoma prevalence surveys in Mongolia (carried out in 1995 and 1997) were treated with YAG laser iridotomy at the time of diagnosis. These patients were re-examined in 1998 . Patency of iridotomy, intraocular pressure (IOP), visual acuity, and gonioscopic findings were recorded. Iridotomy was classified unsuccessful in eyes where further surgical intervention was required or in which there was a loss of visual acuity to $<3 / 60$ from glaucomatous optic neuropathy.
\end{abstract}

Results-164 eyes of 98 subjects were examined. Patent peripheral iridotomies were found in $98.1 \%(157 / 160)$ of eyes that had not undergone surgery. Median angle width increased by two Shaffer grades following iridotomy. Iridotomy alone failed in $3 \%$ eyes with narrow drainage angles and either peripheral anterior synechiae or raised IOP, but normal optic discs and visual fields. However, in eyes with established glaucomatous optic neuropathy at diagnosis iridotomy failed in $47 \%$. None of the eyes with occludable angles that were normal in all other respects, and underwent iridotomy, developed glaucomatous optic neuropathy or symptomatic angle closure within the follow up period.

Conclusions-Nd: YAG laser iridotomy is effective in widening the drainage angle and reducing elevated IOP in east Asian people with primary angle closure. This suggests that pupil block is a significant mechanism causing closure of the angle in this population. Once glaucomatous optic neuropathy associated with synechial angle closure has occurred, iridotomy alone is less effective at controlling IOP. (Br f Ophthalmol 2000;84:1255-1259)

Primary angle closure glaucoma (PACG) probably accounts for half the cases of primary glaucoma worldwide and is the commonest type of glaucoma in eastern Asia. ${ }^{1}$ A prevalence rate of $1.4 \%$ of PACG and $6.5 \%$ of occludable angles was reported in the Mongolian province of Hövsgol in $1995 .^{2}$ A second survey has been carried out in the southern Ömnögobi province to investigate potential methods of screening for angle closure glaucoma. ${ }^{3}$
In Mongolia and other developing countries resources are limited and access to primary eye care is difficult. In many cases it is not practical to examine patients regularly or use topical medication. A "once only" treatment of PACG and primary angle closure (PAC) in the form of laser iridotomy may offer an ideal alternative. Prophylactic treatment of eyes at risk of angle closure may also be worthwhile. The portable Nd:YAG laser is robust, and easy to transport and set up in rural areas. ${ }^{4}$

Laser iridotomy has been shown to be an effective primary therapy for early PACG although advanced cases require further treatment with topical medication or filtration surgery. ${ }^{56}$ Unaffected fellow eyes of patients who have had angle closure have a 50\% risk of developing an episode of angle closure if not treated with prophylactic iridotectomy. ${ }^{78}$ Iridotomy acts by eliminating relative pupil block which is one mechanism underlying the development of angle closure. Non-pupil block mechanisms may also contribute to angle closure in Asians. ${ }^{9-11}$ The relative importance of these factors in different Asian populations, and consequently the degree of benefit conferred by iridotomy, is not clear.

In this population based study we examined the efficacy of YAG laser iridotomy in the management of people with occludable drainage angles, and those with established glaucomatous optic neuropathy in rural Mongolia. Factors that may affect the outcome of iridotomy were evaluated.

\section{Materials and methods}

Ethical approval for this project was obtained from the Mongolian Ministry of Health. During glaucoma prevalence surveys of 1995 and 1997 in the Mongolian provinces of Hövsgöl and Ömnögobi, respectively, subjects with occludable angles were treated with YAG laser iridotomy in the affected eye using a portable laser (Carl Zeiss Visulas II, Carl Zeiss Ltd, Germany). In addition, people with occludable angles referred for assessment by local doctors were also treated with iridotomy. All these people were advised to seek regular follow up with the local ophthalmologist.

\section{SELECTION AND SAMPLING OF SUBJECTS}

Subjects treated with laser iridotomy in 1995 and 1997 were traced and invited for reexamination in 1998. Details of the sampling in the prevalence surveys have been described elsewhere. ${ }^{2}{ }^{3}$ In both surveys the samples were selected randomly from the over 40 year old population. 
CLINICAL ASSESSMENT

Snellen visual acuities were measured on all subjects, and pinhole acuities were measured if less than $6 / 12$. Visual fields were assessed using a static, semiautomated field analyser (Henson 3000, Tinsley Medical Instruments, Newbury, UK). ${ }^{2}$

A slit lamp examination was carried out on every subject, looking for ischaemic sequelae of angle closure and signs of secondary glaucoma. The laser iridotomy site was examined for patency by retroillumination and direct visualisation of structures posterior to the hole. Intraocular pressure (IOP) was measured with a tonometer (Goldmann model, Haag-Streit, Bern, Switzerland). The median of three tonometer readings was recorded as the final IOP for each eye. Gonioscopy was performed with a two mirror Goldmann gonioscopy lens. The width of the irido-trabecular recess was recorded in the four quadrants. The angle was graded occludable or open (see diagnostic criteria). Dynamic (indentation) gonioscopy using either the two mirror Goldmann lens or Sussman four mirror lens was used to assess the presence or absence of peripheral anterior synechiae (PAS) in each quadrant.

All eyes with angles judged not occludable were dilated with tropicamide $1 \%$. The optic disc was examined with the central lens of the Goldmann gonioprism. The vertical cup:disc ratio was measured with the aid of a graticule (measuring eyepiece, Haag-Streit, Bern, Switzerland). The fundus was examined for any other pathology.

All slit lamp examinations at follow up were done by the same observer. Interobserver agreement was measured between the field investigator of this study (WN) and the two investigators on the previous surveys (PF, 1995 and JD, 1997) for measurements of IOP, gonioscopy angle grading, and optic disc assessment. To calculate interobserver agreement measurements were taken by $\mathrm{WN}$ and $\mathrm{PF}$ on 40 eyes of 20 people from a random sample of Chinese Singaporeans who were taking part in a glaucoma survey. WN and JD recorded these measurements on 32 eyes of 16 Mongolian people, some with normal eyes and the remainder who were a mix of normal cases and individuals with occludable angles presenting outside the study. The gonioscopic angle width of the eyes examined for interobserver measurement varied over the range of Shaffer grades $0-4$.

In four subjects follow up examinations were conducted as home visits. In these cases a hand held slit lamp was used for anterior segment examination, gonioscopy was not done and IOP was measured using a Tonopen. A direct ophthalmoscope was used for fundus examination.

\section{DIAGNOSTIC CRITERIA}

An occludable angle was defined as one in which three quarters of the posterior pigmented trabecular meshwork was not visible on viewing with a Goldmann two mirror lens in the primary position of gaze without indentation.
Primary angle closure glaucoma (PACG) was diagnosed in eyes with an occludable angle and glaucomatous optic neuropathy. Evidence of glaucomatous optic neuropathy was defined as a cup:disc ratio (CDR) of $\geqslant 0.7$ or $\geqslant 0.2$ CDR asymmetry. In cases of early structural damage (0.7-0.8 CDR or 0.2 asymmetry) a reproducible visual field defect was required to confirm the diagnosis. In cases with advanced damage ( $\geqslant 0.9$ CDR or $\geqslant 0.3$ asymmetry) perimetric testing was not an absolute requirement in making the diagnosis. PACG was also diagnosed in eyes where it was not possible to visualise the optic disc, but which had ischaemic sequelae of primary angle closure (iris stromal atrophy with sectoral distortion of radially oriented musculature) and visual acuity $<3 / 60$.

Primary angle closure (PAC) (previously termed "latent angle closure glaucoma") was diagnosed in eyes with an occludable angle, normal optic discs and visual fields and any of the following: raised IOP (>19 $\mathrm{mm} \mathrm{Hg})$, PAS, pigment smearing in the superior angle, or sequelae of acute angle closure (iris whorling or glaucomflecken). A positive darkroom/ prone provocation test (rise in IOP $=8 \mathrm{~mm}$ $\mathrm{Hg}$ ) was another finding used to make this diagnosis in 1995. This diagnosis was also made in fellow eyes of eyes with PACG. An eye with an occludable angle but no other abnormality was classified as primary angle closure suspect (PACS); $19 \mathrm{~mm} \mathrm{Hg}$ was used as the "upper limit of normal IOP" in both the original glaucoma surveys ${ }^{2}{ }^{3}$ and in this study. Mean IOP in east Asian populations has been reported as lower than in white people. ${ }^{12}$ The value of $19 \mathrm{~mm} \mathrm{Hg}$ was based on the mean IOP (+ 2 SD) from studies in Chinese and Eskimo populations ${ }^{13}{ }^{14}$ which share a genetic heritage with the people of Mongolia.

Laser peripheral iridotomy was deemed to have failed on its own as the primary treatment, when glaucoma filtering surgery was required to control IOP either between the laser procedure and follow up examination or at the time of follow up. Eyes with a reduction in visual acuity to $<3 / 60$ with no other cause apart from glaucomatous optic disc damage were also classified as failures.

MANAGEMENT OF UNCONTROLLED GLAUCOMA In the six eyes with non-patent or small iridotomies and occludable angles a further YAG laser treatment was given. Subjects with eyes that had evidence of glaucoma and persistently elevated IOP (>19 $\mathrm{mm} \mathrm{Hg}$ ) with a patent iridotomy were advised on management. Filtering surgery in the form of a trabeculectomy was offered and in cases where the subject refused surgery or it was not appropriate (visual loss due to glaucoma was advanced and there was unlikely to be any benefit) topical medication was prescribed.

DATA ANALYSIS

SPSS (Statistical Package for Social Sciences) version 6.1 was used for statistical analysis. McNemar's test was used to assess numbers of eyes before and after iridotomy treatment, in two IOP measurement categories - >19 mm 
Table 1 Age and sex distributions of study populations

\begin{tabular}{llll}
\hline Age (years) & Male & Female & $\begin{array}{l}\text { Male and female (\% of } \\
\text { total) }\end{array}$ \\
\hline Hovsgol (1995) sample & & & \\
\hline $40-49$ & 1 & 3 & $4(10.3)$ \\
$50-59$ & 2 & 7 & $9(23)$ \\
$60-69$ & 3 & 12 & $15(38.5)$ \\
$\geqslant 70$ & 4 & 7 & $11(28.2)$ \\
Total & $10(25.6)$ & $29(74.4)$ & $39(100)$ \\
\hline
\end{tabular}

Ömnögobi (1997) sample

\begin{tabular}{llll}
\hline $40-49$ & 1 & 3 & $4(6.8)$ \\
$50-59$ & 5 & 12 & $17(28.8)$ \\
$60-69$ & 4 & 15 & $19(32.2)$ \\
$\geqslant 70$ & 8 & 11 & $19(32.2)$ \\
Total (\%) & $18(28.2)$ & $41(71.8)$ & $59(100)$ \\
\hline
\end{tabular}

Table 2 Initial diagnosis in eyes treated with laser iridotomy

\begin{tabular}{llll}
\hline Diagnosis & Hövsgol (1995) & Ömnögobi (1997) & Total \\
\hline PACG & $13(20.3 \%)$ & $10(10 \%)$ & $23(14 \%)$ \\
PAC & $44(68.8 \%)$ & $23(23 \%)$ & $67(40.9 \%)$ \\
PACS & $7(10.9 \%)$ & $67(67 \%)$ & $74(45.1 \%)$ \\
Total & $64(100 \%)$ & $100(100 \%)$ & $164(100 \%)$ \\
\hline
\end{tabular}

PACG $=$ primary angle closure with glaucomatous optic neuropathy.

$\mathrm{PAC}=$ primary angle closure.

PACS $=$ primary angle closure suspect.

$\mathrm{Hg}$ and $\leqslant 19 \mathrm{~mm} \mathrm{Hg}$. Univariate and multivariate logistic regression analysis was used to assess predictors for outcome.

\section{Results}

Sixty four subjects were treated with YAG laser iridotomy in 1995 and 84 subjects were treated in 1997. Re-examination was possible on 39 (64 eyes) of the 64 subjects treated in 1995 $(60.9 \%)$, and 59 (100 eyes) of 84 treated in $1997(70.2 \%)$. In 1995, 16 patients were referred from outside the sampling frame of the survey whereas in 1997 only five cases were outside the sampling frame. The median follow up time was 35 months (range 33-37) for the 1995 group and 11 months (range 10-11) for the 1997 group. The age and sex distributions of the subjects examined are shown in Table 1 and the distribution of diagnosis by eye (at initial examination) is set out in Table 2 .

During the follow up interval (between laser iridotomy and re-examination) four eyes had glaucoma surgery. These are excluded from analysis of slit lamp findings including patency of iridotomy and gonioscopy. They are classified as failures in the analysis of overall outcome of iridotomy and as having IOP >19 $\mathrm{mm} \mathrm{Hg}$ in the analysis of effect of iridotomy on IOP.

Patent iridotomies were present in 157 of $160(98.1 \%)$ eyes treated (excluding the four eyes mentioned above). In two eyes of the 1995 group and one of the 1997 group the iridotomies had closed over. In three eyes of the 1997 group the angles were still occludable or borderline occludable. It had been recorded in three of these six cases at the time of treatment that the iridotomy holes were small.

Thirteen of $164(7.9 \%)$ eyes treated were classed as failures at follow up; 10 eyes from the 1995 group and three of the 1997 group. Of the 13 eyes, four had been operated on with either surgical iridectomy or trabeculectomy during the follow up period, five were referred for trabeculectomy at the time of follow up, and four had end stage glaucoma with "light perception" or worse visual acuity in the affected eye. All but two of the eyes classified as failures had evidence of glaucomatous optic neuropathy before iridotomy. The two remaining cases had PAC, one of which also had pseudoexfoliation. Both these PAC cases had elevated IOPs at follow up despite open angles and no PAS. Apart from two cases which were classified as failures, none of the patients was using topical medication at follow up. The failure rate in PACG eyes was $11 / 23(47.8 \%)$ and in PAC eyes was $2 / 67(3 \%)$.

None of the 74 PACS eyes that had been treated with iridotomy had developed glaucoma or ischaemic sequelae of primary angle closure between treatment and follow up.

INTRAOCULAR PRESSURE

Twenty seven eyes had an IOP recorded as $>19$ $\mathrm{mm} \mathrm{Hg}$ before iridotomy. Of these, 15 (55.5\%) had IOP $\leqslant 19 \mathrm{~mm} \mathrm{Hg}$ at follow up on no medication (McNemar's test for matched pairs $\chi^{2}=15, \mathrm{p}<0.01$ ) (Table 3). In $8 / 27$ eyes the IOP was $>19 \mathrm{~mm} \mathrm{Hg}$ at follow up and in $4 / 27$ eyes, as mentioned above, glaucoma surgery was performed during the follow up period. Surgery was required in these four cases as laser iridotomy had failed to control the IOP. Therefore for the purposes of this analysis they are defined as having IOP $>19 \mathrm{~mm} \mathrm{Hg}$ at follow up. Extent of change in IOP after iridotomy is demonstrated in Figure 1 (excluding the four operated eyes).

\section{GONIOSCOPY}

In all, 148 of 151 (98\%) iridotomy treated eyes had angles that were no longer classified as occludable (excluding the four operated eyes, eight eyes examined at home visits, and one eye with dense corneal scarring). Of the three eyes with angles that remained occludable one had a non-patent iridotomy, another had a small iridotomy, and the third had extensive PAS covering the trabecular meshwork.

Table 3 Pre and post laser intraocular pressures (IOP) greater than $19 \mathrm{~mm} \mathrm{Hg}$

\begin{tabular}{|c|c|c|c|c|c|c|}
\hline \multirow[b]{2}{*}{$I O P>19 \mathrm{~mm} \mathrm{Hg}$} & \multicolumn{3}{|c|}{$P A C G$ (no of eyes) } & \multicolumn{3}{|c|}{$P A C$ (no of eyes) } \\
\hline & 1995 group & 1997 group & Total & 1995 group & 1997 group & Total \\
\hline Before laser & $9 / 13$ & $7 / 10$ & $16 / 23$ & $8 / 44$ & $3 / 23$ & $11 / 67$ \\
\hline After laser & $7 / 13$ & $3 / 10$ & $10 / 23$ & $2 / 44$ & $0 / 23$ & $2 / 67$ \\
\hline \multicolumn{7}{|c|}{$\begin{array}{l}\text { McNemar's test for matched pairs } \\
\chi^{2}=15, \mathrm{p}<0.01\end{array}$} \\
\hline
\end{tabular}

PACG = primary angle closure glaucoma with glaucomatous optic neuropathy.

$\mathrm{PAC}=$ primary angle closure. 


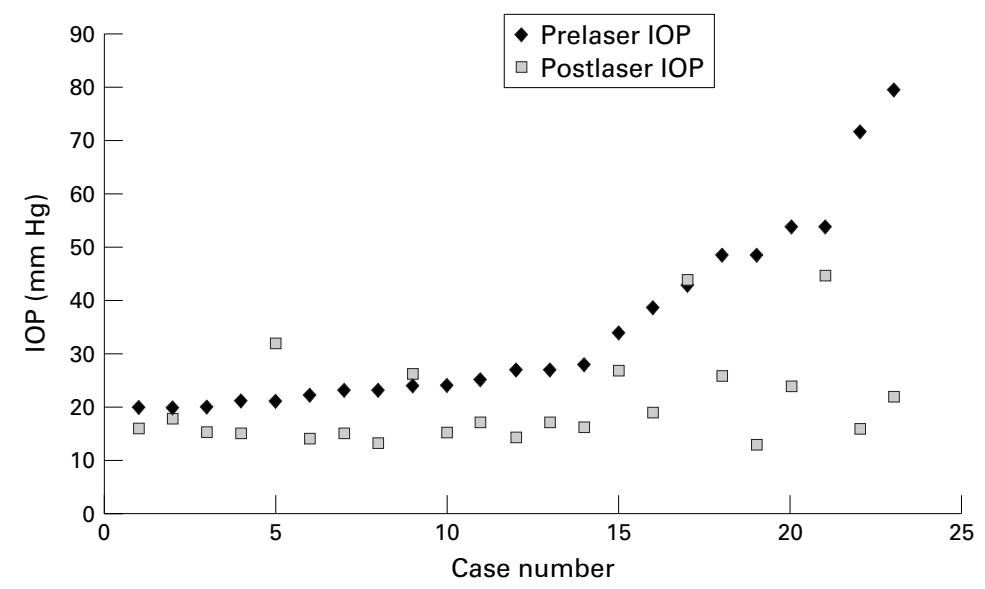

Figure 1 Pre and post iridotomy intraocular pressures (IOP) in eyes with IOP $>19 \mathrm{~mm}$ $\mathrm{Hg}$ before iridotomy (excluding four eyes that underwent glaucoma surgery during follow up interval).

Table 4 Relation between pre-iridotomy examination findings and outcome of iridotomy in eyes with diagnosis of $P A C$ or $P A C G$

\begin{tabular}{|c|c|c|}
\hline \multirow[b]{2}{*}{ Pre-laser examination findings } & \multicolumn{2}{|c|}{ Outcome of iridotomy } \\
\hline & Failure (no of eyes) & Stable (no of eyes) \\
\hline$(\underline{C D R} \geqslant 0.8)+($ PAS 3-4 $)+($ IOP $>19)$ & 7 & 1 \\
\hline$(\overline{\mathrm{CDR}} \geqslant 0.8)+(\overline{\mathrm{PAS} 3-4})+(\overline{\mathrm{IOP} \leqslant 19})$ & 1 & 1 \\
\hline$(\overline{\mathrm{CDR}} \geqslant 0.8)+(\overline{\mathrm{PAS} \mathrm{0}-2})+(\mathrm{IOP}>19)$ & $1^{\star}$ & 1 \\
\hline$(\overline{\mathrm{CDR}<0.8)}+(\underline{\mathrm{PAS}} 3-4)+(\overline{\mathrm{IOP}>19})$ & 3 & 5 \\
\hline$(\mathrm{CDR} \geqslant 0.8)+(\mathrm{PAS} 0-2)+(\mathrm{IOP} \leqslant 19)$ & 0 & 1 \\
\hline$(\overline{\mathrm{CDR}<0.8)}+(\mathrm{PAS} 3-4)+(\mathrm{IOP} \leqslant 19)$ & 0 & 7 \\
\hline$(\mathrm{CDR}<0.8)+($ PAS 0-2 $)+(\mathrm{IOP}>19)$ & $1 \dagger$ & 7 \\
\hline$(\mathrm{CDR}<0.8)+(\mathrm{PAS} 0-2)+(\overline{\mathrm{IOP} \leqslant 19})$ & 0 & 45 \\
\hline Total & 13 & 68 \\
\hline
\end{tabular}

${ }^{\star}$ Pseudoexfoliation; $\nmid$ mixed mechanism glaucoma; PAS = number of angle quadrants containing peripheral anterior synechiae; IOP $=$ intraocular pressure $(\mathrm{mm} \mathrm{Hg}) ; \mathrm{CDR}=$ cup:disc ratio.

Numbers after PAS (peripheral anterior synechiae) indicate number of angle quadrants containing PAS.

Table 5 Logistic regression analysis of predictive findings for outcome of failure with iridotomy

\begin{tabular}{llll}
\hline Factor & OR & $95 \% C I$ & Significance \\
\hline Univariate analysis & 7.78 & $1.87-32.33$ & $\mathrm{p}=0.005$ \\
PAS (3-4) & 26.43 & $5.56-125.62$ & $\mathrm{p}<0.0001$ \\
CDR $\geqslant 0.8$ & 31.7 & $3.7-266.93$ & $\mathrm{p}=0.002$ \\
IOP $>19$ & $0.38-15.97$ & $\mathrm{p}=0.34$ \\
Multivariate analysis & & $2.28-99.29$ & $\mathrm{p}=0.005$ \\
PAS (3-4) & 2.47 & $1.85-212.49$ & $\mathrm{p}=0.01$ \\
CDR $\geqslant 0.8$ & 15.04 & 19.8 & IOP $>19$
\end{tabular}

PAS = number of quadrants containing perpheral anterior synechiae.

$\mathrm{CDR}=$ cup:disc ratio.

$\mathrm{IOP}=$ intraocular pressure $(\mathrm{mm} \mathrm{Hg})$.

$\mathrm{OR}=$ odds ratio or four quadrants containing PAS, and IOP $>19 \mathrm{~mm} \mathrm{Hg}$ at the pre-laser examination (Table 4). Results of univariate and multivariate logistic regression analysis of predictive factors for a failure outcome after iridotomy are shown in Table 5. The nine fellow eyes classified as PAC but with occludable angles only are excluded from this analysis. The adjusted odds ratios are lower in the multiple regression model and in the case of PAS do not reach significance, demonstrating the relation between the three factors. The results of this analysis should be interpreted with caution in view of the large confidence intervals which are due to the small number of eyes in the failure outcome group.

\section{INTEROBSERVER AGREEMENT}

Good agreement between the field investigator on this study (WN) and the investigators of the 1995 study (PF) and the 1997 study (JD) for gonioscopic Shaffer grading was achieved (weighted kappa $=0.62,0.73$ respectively). The mean difference in IOP measurements between WN and PF was $0.75 \mathrm{~mm} \mathrm{Hg}$ (SD 2.74) with WN reading lower measurements. Good agreement was achieved for optic disc grading (weighted kappa $=0.84, \mathrm{WN}$ and $\mathrm{PF}$ ).

\section{Discussion}

The outcome of the cases in this study was related to the stage of disease at diagnosis. Forty seven per cent of eyes with glaucomatous optic neuropathy were classed as treatment failures on grounds that IOP was poorly controlled or visual acuity had deteriorated due to progression of glaucoma. Three per cent of PAC eyes were found to have glaucomatous optic neuropathy at follow up, but there were other features such as pseudoexfoliation to explain a poor prognosis. None of the PACS eyes suffered symptomatic angle closure or glaucomatous optic neuropathy. It appears that once extensive synechial angle closure and glaucomatous optic neuropathy have developed, an iridotomy will be less likely to reduce IOP and protect visual function than in eyes treated at an earlier stage. However, it must be acknowledged that this finding could be explained by the phenomenon of "lead time bias". This form of bias can result in an apparent increase in survival or, in the case of glaucoma, increased time to loss of visual function in a case detected and treated at an early stage of the disease process. The real time from incidence of disease to visual function loss or blindness in such a case may be no different from that of a case detected at a more advanced stage. ${ }^{15}$ Longer follow up or a randomised controlled trial would be required to overcome the possible influence of this bias.

Long term patency rates of YAG laser iridotomy were very good in these dark Asian irides in line with other studies in white and Afro-Caribbean eyes. ${ }^{16-18}$ Closure, or reduced effectiveness of the iridotomy, seemed to be related to a documented "small" initial hole.

Our results show normalisation of IOP without medication in $55.5 \%(15 / 27)$ of eyes with elevated pressure before iridotomy. None of of PAC or PACG that were classified as failures seven had a combination of $\mathrm{CDR} \geqslant 0.8$, three 
the eyes with an IOP $\leqslant 19 \mathrm{~mm} \mathrm{Hg}$ before treatment had elevated IOP at follow up. Other studies have shown rates of IOP normalisation ranging from $9 \%$ without medication and $51 \%$ with medication to $90 \%$ in a mixture of cases with and without maintenance medication. ${ }^{5}{ }^{19}$

CDR $\geqslant 0.8$, PAS involving three to four angle quadrants, and IOP $>19 \mathrm{~mm} \mathrm{Hg}$ at diagnosis were all significantly associated with future failure of iridotomy. Table 4 shows that in eyes where two of these factors were present, approximately half were controlled with iridotomy, although there were small numbers of eyes with $\mathrm{CDR} \geqslant 0.8$ with one other factor; $15 / 16$ eyes with only one of these factors had a successful outcome after iridotomy. Eyes with all three factors present appear to be at highest risk of failure after iridotomy. Quigley found no association between any examination findings before treatment and outcome of argon laser iridotomy, ${ }^{20}$ but several studies demonstrate a relation between the extent of angle closure by PAS and failure of iridotomy to control IOP and progression of glaucoma. ${ }^{5621}$ Iridectomy or iridotomy is less effective in eyes with glaucomatous visual field loss and further surgical or medical treatment is often required to control IOP. ${ }^{22} 23$

In the absence of sophisticated resources, $\mathrm{CDR}$ is probably more useful than visual fields in predicting outcome of treatment. Visual field testing in a rural population is fraught with problems related to reliability and the need for multiple tests to achieve a baseline result. Our results suggest that in PACG eyes with advanced optic disc damage and three to four quadrants of PAS, trabeculectomy would be a better option than iridotomy. However, we do not know the success rate of filtering surgery in this population. It is likely that early detection of eyes at risk of angle closure will increase the probability of success of an iridotomy.

Despite the subjective nature of gonioscopy we have demonstrated good agreement between observers. It may be more difficult to reliably assess PAS in the presence of an occludable angle at initial examination. PAS were reassessed a day after iridotomy when the angle had widened.

The development of new PAS in the presence of a patent iridotomy suggests that irido-trabecular apposition is still occurring. IOP rises are known to occur in some eyes with patent iridectomies subjected to the dark prone provocative tests. ${ }^{9}$ As pupil block is eliminated by an iridotomy, ciliolenticular block or peripheral iris crowding are presumed to play a part in these pressure rises. Despite this, it does appear that pupil block is the main mechanism in the development of angle closure in the Mongolian population. This is demonstrated by reduction in IOP, widening of the angle, and recorded regression of PAS in some of the cases of PACG and PAC.

In summary, this is the first comprehensive study of the outcome of peripheral iridotomy in Mongolia, and may have relevance to neighbouring populations. It is documented that glaucoma and, particularly, PACG are a major cause of blindness in the east Asian region. ${ }^{24}$
This fact demands that we investigate strategies for the prevention of PACG. Laser peripheral iridotomy appears to offer a simple, low risk method of achieving this goal. However the appropriate timing for intervention remains uncertain.

To answer this question, we have begun a randomised controlled trial of screening for primary angle closure in Mongolia using bilateral laser peripheral iridotomy as a prophylactic treatment.

This study was funded by the National Lottery Charities Board, through Fight for Sight with additional funding from the International Glaucoma Association, the British Council for the Prevention of Blindness, and the Danish Association for the Blind. The YAG laser used in this study was donated by the Velux Foundation, Copenhagen. We thank Pak Sang Lee, MSc, BSc, technical adviser, Institute of Ophthalmology, Catey Bunce, MSc, medical statisMD, Dr Med, Department of Ophthalmology, Hillerød Hospital, Hillerød, Denmark.

Proprietary interests: None.

1 Quigley HA. Number of people with glaucoma worldwide. Br f Ophthalmol 1996;80:389-93.

2 Foster PJ, Baasanhu J, Alsbirk PH, et al. Glaucoma in Mongolia-a population-based survey in Hövsgöl Province, northern Mongolia. Arch Ophthalmol 1996;114:123541 .

3 Devereux JG, Foster PJ, Baasanhu J, et al. Anterior chamber depth measurement as a screening tool for primary angleclosure in an East Asian population. Arch Ophthalmol 2000;118:257-63.

4 Robin AL, Arkell S, Gilbert SM. Q-Switched neodymiumYAG laser iridotomy. A field trial with a portable laser system. Arch Ophthalmol 1986;104:526-30.

5 Salmon JF. Long-term intraocular pressure control after Nd-YAG laser iridotomy in chronic angle-closure glaucoma. F Glaucoma 1993;2:291-6.

6 Yamamoto T, Shirato S, Kitazawa Y. Treatment of primary angle-closure glaucoma by argon laser iridotomy: a angle-closure glaucoma by argon laser iridotomy:

7 long-term follow-up. Fpn f Ophthalmol $1985 ; 29: 1-12$. second eye in angle-closure glaucoma. Trans Ophthalmol Soc UK 1977;97:189-91.

8 Lowe RF. Acute angle-closure glaucoma. The second eye: an analysis of 200 cases. Br F Ophthalmol 1962;46:641-50.

9 Hung PT, Chou LH. Provocation and mechanism of angleclosure glaucoma after iridectomy. Arch Ophthalmol 1979; 97:1862-4.

10 Lowe RF, Ritch R. Angle-closure. Clinical types. In: Ritch $\mathrm{R}$, Shields MB, Krupin T. eds. The glaucomas. St Louis, MO: CV Mosby; 1989:839-53.

11 Sakuma T, Sawada A, Yamamoto T, et al. Appositional angle closure in eyes with narrow angles: an ultrasound closure in eyes with narrow angles: an ulta

12 Shiose Y. Intraocular pressure. New perspectives. Surv Ophthalmol 1990;34:413-35.

$13 \mathrm{Hu}$ Z, Zhao ZL, Dong FT. An epidemiological investigation of glaucoma in Beijing and Shun-yi county. Chin $f$ Ophthalmol 1989;25:115-8.

14 Arkell SM, Lightman DA, Sommer A, et al. The prevalence of glaucoma among eskimos of Northwest Alaska. Arch Ophthalmol 1987;105:482-5.

15 Wormald R. Screening in ophthalmology. In: Johnson GJ, Minassian DC, Weale R, eds. The epidemiology of eye disease. London: Chapman and Hall, 1998:83-100.

16 Schwartz LW, Moster MR, Spaeth GL, et al. NeodyniumYAG laser iridectomies in glaucoma associated with closed or occludable angles. Am fo Ophthalmol 1986;102:41-4.

17 Canning CR, Capon MRC, Sherrard ES, et al. Neodymium: YAG laser iridotomies-short-term comparison with capYAG laser iridotomies-short-term comparison with cap-
sulotomies and long-term follow-up. Graefes Arch Clin Exp Ophthalmol 1988;226:49-54.

18 Del-Priore LV, Robin AL, Pollack IP. Neodymium:YAG and argon laser iridotomy. Long-term follow-up in a prospective, randomized clinical trial. Ophthalmology 1988;95: 1207-11.

19 Tomey KF, Traverso C, Shammas IV. Neodymium-YAG laser iridotomy in the treatment and prevention of angle closure glaucoma. A review of 373 eyes. Arch Ophthalmol 1987;105:476-81.

20 Quigley HA. Long-term follow-up of laser iridotomy. Ophthalmology 1981;88:218-24

21 Kim YY, Jung HR. Dilated miotic-resistant pupil and laser iridotomy in primary angle-closure glaucoma. Ophthalmologica 1997;211:205-8.

22 Gelber EC, Anderson DR. Surgical decisions in chronic angle-closure glaucoma. Arch Ophthalmol 1976;94:1481-4.

3 Richardson P, Cooper RL. Laser iridotomy. Aust NZ 7 Ophthalmol 1987;15:119-23.

24 Baasanhu J, Johnson GJ, Burendei G, et al. Prevalence and causes of blindness and visual impairment in Mongolia: a causes of blindness and visual impairment in Mongolia: a
survey of populations aged 40 years and older. Bull World Health Organ 1994;72:771-6. 